\title{
CYTOTOXICITY OF COLCHICINE DERIVATIVES IN PRIMARY CULTURES OF HUMAN HEPATOCYTES
}

\author{
Zdenek Dvorak $^{\text {a*}}$, Jitka Ulrichovaa ${ }^{\text {a }}$ Roland Weyhenmeyer ${ }^{\mathrm{b}}$, Vilim Simanek ${ }^{\mathrm{a}}$ \\ a Institute of Medical Chemistry and Biochemistry, Faculty of Medicine and Dentistry Palacký University Olomouc, \\ Hnèvotínská 3, 77515 OLOMOUC, Czech Republic \\ ${ }^{b}$ Pfarrer-Korner-Strase 8, D-51429 Bergisch Gladbach, Germany \\ e-mail:moulin@email.cz
}

Received: April 19, 2007; Accepted: May 10, 2007

Key words: Colchicine derivatives/Toxicity/Human hepatocytes

Background: Colchicine has been used to treat gout for centuries. However, owing to its toxicity it displays a variety of side effects. The replacement of colchicine by less toxic but still active derivatives would solve this drawback.

Aim: The aim of this study was to examine the cytotoxicity of 17 colchicine derivatives.

Methods: Primary cultures of human hepatocytes were the model of choice. Prior to testing, we measured the biochemical parameters of liver donors and the toxicological response of the hepatocytes cultures. For toxicity testing, cells were treated for $24 \mathrm{~h}$ with tested compounds in concentrations 1-100 $\mu \mathrm{M}$. We monitored lactate dehydrogenase (LDH) leakage into the medium, mitochondrial activity (MTT test) and secretion of albumin.

Results: Our data show that LDH and MTT were less sensitive parameters compared to albumin secretion for monitoring the toxicity of colchicine derivatives. Compounds with lower antimitotic activity displayed lowered toxicity.

Conclusion: Since human hepatocytes in culture are quiescent cells, they are not as susceptible to tropolone alkaloids as proliferating cells. Screening for colchicine derivatives with lowered cytotoxicity revealed that 10-O-demethylated compounds might be the substances of choice.

\section{INTRODUCTION}

Colchicine has been used to treat gout for centuries. It has also medicinal applications in the treatment of some auto-immune and dermatologic disorders ${ }^{1,2}$. However, owing to its toxicity it displays a variety of side effects ${ }^{1}$. Replacement of colchicine by less toxic but still active derivatives would solve this drawback ${ }^{3}$. In addition, structural modification to colchicine could produce compounds with novel pharmacological activity.

At a molecular level, colchicine has various effects on tubulin, the major one being a change in the secondary structure of the protein caused by binding to a high affinity site on the tubulin heterodimer. This binding induces partial unfolding of the secondary structure of $\beta$ tubulin at the carboxy terminus ${ }^{4}$. This unfolding disrupts the protein regions necessary for microtubule formation ${ }^{5}$. Microtubule disarray leads to a variety of consequences at the molecular level ${ }^{6-8}$.

The aim of this study was to examine the cytotoxicity of 17 colchicine derivatives. These were newly synthesized and are not commercially available. The cytotoxicity assessment was performed on primary cultures of human hepatocytes, as the most suitable model for biochemical and toxicological studies on hepatic metabolism in $\operatorname{man}^{9,10}$.

\section{MATERIALS AND METHODS}

\section{Chemicals}

Cell culture media, supplements and foetal calf serum were purchased from Sigma (St. Louis, MO, USA). All other chemicals and reagents were of the highest quality commercially available.

\section{Tested compounds}

The characteristics of the tested compounds are listed in Table 1. The structures of tested compounds are shown in Fig 1. Compounds A0, A11, A12, A13, A14, A15 were from Collection of Alkaloids, Institute of Medical Chemistry and Biochemistry, Faculty of Medicine, Palacky University, Olomouc, Czech Republic. Compounds purity was checked by HPLC ${ }^{11}$. Compounds A1, A2, A3, A4, A5, A6, A7, A8, A9, A10, A16, A17 were from MADAUS AG, Cologne, Germany.

\section{Primary cultures of human hepatocytes}

Liver samples were obtained from multi-organ donors. The tissue acquisition protocol was in accordance with the requirements issued by the Ethics Commission of the Faculty Hospital Olomouc. The characterization of liver donors is shown in Table 2. Hepatocytes were isolated according to a published protocol ${ }^{12}$. The cells were plated on collagen coated culture dishes using cell density $14 \mathrm{x}$ $10^{4}$ cells $/ \mathrm{cm}^{2}$. The culture medium used was as described ${ }^{13}$ 

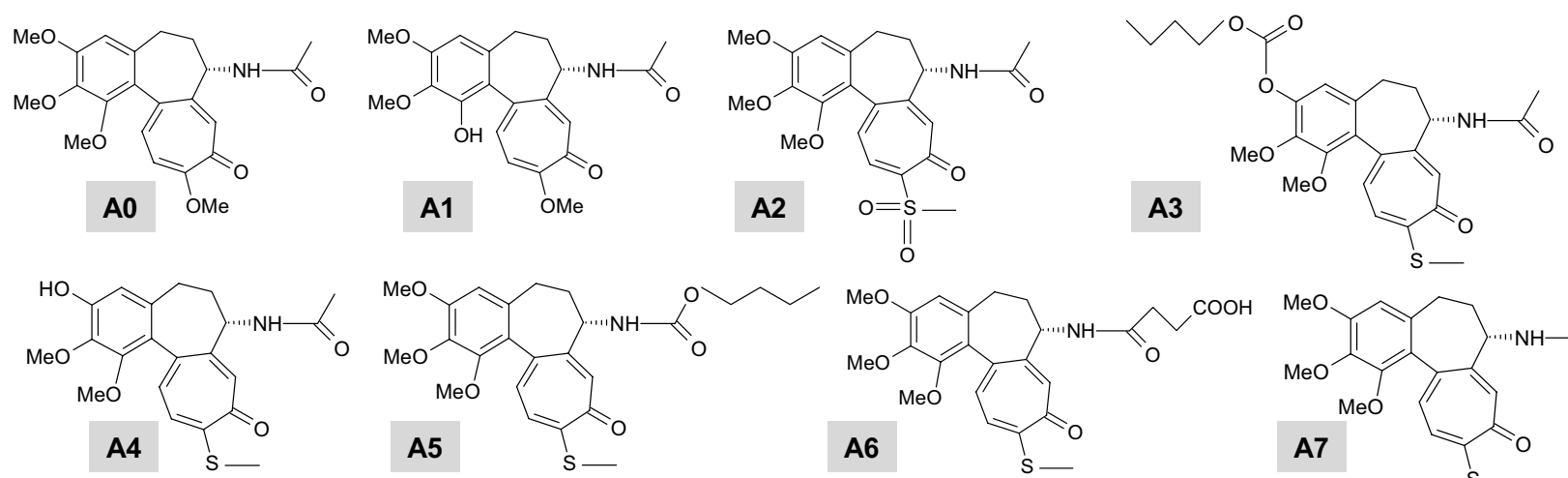<smiles>C=C(C)N[C@H]1CCc2cc(OC)c(OC)c(OC)c2-c2ccc(SC)c(=O)cc21</smiles><smiles>CCCCOC=O</smiles><smiles>COCC(=O)N[C@H]1CCc2cc(OC)c(OC)c(OC)c2-c2ccc(SC)c(=O)cc21</smiles><smiles></smiles><smiles>COc1cc2c(c(O)c1OC)-c1ccc(SC)c(=O)cc1[C@@H](NC(C)=O)CC2</smiles><smiles>COc1cc2c(c(OC)c1OC)-c1ccc(SC)c(=O)cc1[C@@H](NC(=O)C(F)(F)F)CC2</smiles><smiles>COc1cc2c(c(OC)c1OC)-c1ccc(O)c(=O)cc1[C@@H](N)CC2</smiles><smiles>COc1c(O)cc2c(c1OC)-c1ccc(OC)c(=O)cc1[C@@H](NC(C)=O)CC2</smiles><smiles>COc1ccc2c(c1)-c1cc(OC)c(=O)ccc1[C@@H](NC(C)=O)CC2</smiles><smiles>CN[C@H]1CCc2cc(OC)c(OC)c(OC)c2-c2ccc(OC)c(=O)cc21</smiles><smiles>COc1cc2c(c(OC)c1OC)-c1ccc(N)c(=O)cc1[C@@H](NC(C)=O)CC2</smiles><smiles>CNc1ccc2c(cc1=O)[C@H](NC(C)=O)CCc1cc(OC)c(OC)c(OC)c1-2</smiles>

Fig. 1. Chemical structures of colchicine derivatives.

enriched for plating with $5 \%$ foetal calf serum $(\mathrm{v} / \mathrm{v})$. The medium was exchanged for a serum-free medium four to six hours later and the culture was allowed to stabilize for an additional $24 \mathrm{~h}$. Cultures were maintained at $37^{\circ} \mathrm{C}$ in $5 \% \mathrm{CO}_{2}$ humidified incubator.

\section{Cytotoxicity assessment}

Hepatocytes were incubated $24 \mathrm{~h}$ with the tested compounds. In parallel, cultures were treated with dimethylsulfoxide (DMSO) and 1\% v/v Triton X-100 to assess the minimal and maximal cell damage, respectively. Lactate dehydrogenase (LDH) leakage into the medium and MTT assay were measured as the indicators of membrane damage and viability, respectively ${ }^{14,15}$. Albumin secretion in the medium was monitored by radio-immune assay (ALBUMIN RIA kit; IMMUNOTECH a.s., Prague; cat 3251). Cells treated with Triton X-100 represented $100 \%$ LDH activity in the medium; cells treated with DMSO represented $100 \%$ metabolic activity in the MTT assay and $100 \%$ of functionality in albumin secretion assay. The response of primary cultures to Triton X-100 (LDH) is shown in Table 3.

\section{RESULTS AND DISCUSSION}

\section{Characterization of liver donors}

Liver donors were subjected to virological and biochemical testing prior to the surgery. All donors involved in this report were negative for HIV (human immune-deficiency virus), HCV (hepatitis C virus), EBV (Epstein-Barr virus) and CMV (cytomegalovirus). We also monitored biochemical parameters; i.e. ALT (alanine aminotransferase), AST (aspartate aminotransferase), ALP (serum alkaline phophatase) and $\mathrm{Bi}$ (serum bilirubin). Information on the sex, age and biochemical parameters of liver donors are shown in Table 2. We observed no clear relation between biochemical parameters, age and sex of the donors and the quality of liver specimens or freshly isolated hepatocytes.

\section{Characterization of primary human hepatocytes}

Following the isolation of human hepatocytes we determined their viability using the Trypan Blue exclusion test (Table 3). Cultures were allowed to stabilize for 24 h. Thereafter, we assessed the "toxicological range - TR" of the cultures, i.e. the responsiveness of the cultures membrane damage by Triton-X. TR was calculated as the ratio of $\mathrm{LDH}$ activity in the medium released by cells 
Table 1. The list of colchicine derivatives.

\begin{tabular}{|c|l|c|c|c|}
\hline $\mathrm{Nb}$ & \multicolumn{1}{|c|}{ Compound } & Abbr. & $\mathrm{Mw}$ & Summar formula \\
\hline 1 & colchicine & $\mathrm{A} 0$ & 399.44 & $\mathrm{C}_{22} \mathrm{H}_{25} \mathrm{NO}_{6}$ \\
2 & 1-demethylcolchicine & $\mathrm{A} 1$ & 385.42 & $\mathrm{C}_{21} \mathrm{H}_{23} \mathrm{NO}_{6}$ \\
3 & 10-methylsulphonylcolchicine & $\mathrm{A} 2$ & 447.51 & $\mathrm{C}_{22} \mathrm{H}_{25} \mathrm{NO}_{7} \mathrm{~S}$ \\
4 & 3-butoxycarbonyl-3-demethylthiocolchicine & $\mathrm{A} 3$ & 501.61 & $\mathrm{C}_{26} \mathrm{H}_{31} \mathrm{NO}_{7} \mathrm{~S}$ \\
5 & 3-demethylthiocolchicine & $\mathrm{A} 4$ & 401.49 & $\mathrm{C}_{21} \mathrm{H}_{23} \mathrm{NO}_{5} \mathrm{~S}$ \\
6 & $N$-butoxycarbonyl- $N$-deacetylthiocolchicine & A5 & 473.59 & $\mathrm{C}_{25} \mathrm{H}_{31} \mathrm{NO}_{6} \mathrm{~S}$ \\
7 & $N$-deacetyl- $N$-succinylthiocolchicine & A6 & 473.55 & $\mathrm{C}_{24} \mathrm{H}_{27} \mathrm{NO}_{7} \mathrm{~S}$ \\
8 & $N$-deacetyltrifluoracetyl-thiocolchicine & A7 & 469.48 & $\mathrm{C}_{22} \mathrm{H}_{22} \mathrm{~F}_{3} \mathrm{NO}_{5} \mathrm{~S}$ \\
9 & $N$-ethoxyacetyl- $N$-deacetylthiocolchicine & A8 & 459.57 & $\mathrm{C}_{24} \mathrm{H}_{29} \mathrm{NO}_{6} \mathrm{~S}$ \\
10 & $N$-ethoxycarbonyl- $N$-deacetylthiocolchicine & A9 & 445.54 & $\mathrm{C}_{23} \mathrm{H}_{27} \mathrm{NO}_{6} \mathrm{~S}$ \\
11 & thiocolchicine & A10 & 415.51 & $\mathrm{C}_{22} \mathrm{H}_{25} \mathrm{NO}_{5} \mathrm{~S}$ \\
12 & 10-demethylcolchicine & A11 & 385.42 & $\mathrm{C}_{21} \mathrm{H}_{23} \mathrm{NO}_{6}$ \\
13 & $N$-deacetylcolchiceine & A12 & 343.48 & $\mathrm{C}_{19} \mathrm{H}_{21} \mathrm{NO}_{5}$ \\
14 & 3-demethylcolchicine & A13 & 385.42 & $\mathrm{C}_{21} \mathrm{H}_{23} \mathrm{NO}_{6}$ \\
15 & Colchicoside & A14 & 547.44 & $\mathrm{C}_{27} \mathrm{H}_{34} \mathrm{NO}_{12}$ \\
16 & Demecolcine & A15 & 371.43 & $\mathrm{C}_{21} \mathrm{H}_{25} \mathrm{NO}_{5}$ \\
17 & Colchiceineamide & A16 & 384.44 & $\mathrm{C}_{21} \mathrm{H}_{24} \mathrm{~N}_{2} \mathrm{O}_{5}$ \\
18 & Azacolchicine & A17 & 398.46 & $\mathrm{C}_{22} \mathrm{H}_{26} \mathrm{~N}_{2} \mathrm{O}_{5}$ \\
\hline
\end{tabular}

Table 2. Characterization of liver donors.

\begin{tabular}{|c|c|c|c|c|c|c|}
\hline Donor & Sex & Age & $\begin{array}{c}\text { ALT } \\
{\left[\mu \mathrm{kat.} .^{-1}\right]}\end{array}$ & $\begin{array}{c}\text { AST } \\
{\left[\mu \mathrm{kat} . .^{-1}\right]}\end{array}$ & $\begin{array}{c}\text { ALP } \\
{\left[\mu \mathrm{kat} .1^{-1}\right]}\end{array}$ & $\begin{array}{c}\text { Bi } \\
{\left[\mu \mathrm{mol} . .^{-1}\right]}\end{array}$ \\
\hline LH-7 & M & 38 & 0.64 & 0.32 & n.d. & 5.0 \\
LH-8 & M & 56 & 0.28 & 0.31 & n.d. & 6.1 \\
LH-9 & M & 73 & 0.61 & 0.31 & n.d. & n.d \\
LH-10 & F & 15 & 0.79 & 2.72 & 1.83 & 6.0 \\
LH-11 & M & 25 & 0.60 & 1.42 & 0.82 & 15.0 \\
\hline
\end{tabular}

ALT $=$ serum alanin aminotransferase $;$ AST $=$ serum aspartate aminotransferase; $\mathrm{ALP}=$ serum alkaline phophatase $; \mathrm{Bi}=$ serum bilirubin

challenged with Triton-X over the LDH activity in the medium of control cells $\left(\mathrm{TR}=\mathrm{LDH}_{\text {Triton }} / \mathrm{LDH}_{\text {Control }}\right)$. The toxicological range spanned between cca 2.5 - 11; the differences were more due to the variability of LDH activity released by control cells than to the effects of Triton-X (Table 3).

\section{Cytotoxicity of tested compounds}

To examine the cytotoxicity of tested compounds in primary cultures of human hepatocytes, we measured the following parameters: (i) lactate dehydrogenase activity in culture medium as an indicator of cell membrane damage; (ii) MTT test as the marker of mitochondrial activity; (iii) secretion of albumin into the medium to monitor hepatospecific functions.

Increased activity of $\mathrm{LDH}$ in the medium was observed in cultures incubated with compounds A0, A2, A13 and A15 in concentrations as high as $100 \mu \mathrm{M}$ (Table 4). Similar results were obtained when monitoring the mitochondrial activity of hepatocytes - MTT (Table 5). Tropolone alkaloids are highly toxic compounds. Hence, it seems that LDH and MTT are not optimal and sensitive parameters for monitoring the cytotoxicity of these compounds. This may be due to: (i) Cell membrane and mitochondria are not primary targets for tropolone alkaloids within the cell; (ii) Tropolone alkaloids inhibit 
Table 3. Characterization of primary human hepatocytes. Viability of freshly isolated hepatocytes was assayed by Trypan blue staining. Cells treated with Triton X-100 represented $100 \%$ LDH activity in the medium ( $100 \%$ damage); cells treated with DMSO represented basal activity ( $0 \%$ damage). Toxicological range was calculated as $\mathrm{LDH}_{\text {triton }} / \mathrm{LDH}_{\text {control }}$.

\begin{tabular}{|c|c|c|c|c|}
\hline Liver & Viability & LDH - control $\left[\mu \mathrm{kat} . \mathrm{L}^{-1}\right]$ & $\mathrm{LDH}$ - triton $\left[\mu \mathrm{kat} . \mathrm{L}^{-1}\right]$ & $\begin{array}{l}\text { Toxicological range } \\
\mathrm{LDH}_{\text {triton }} / \mathrm{LDH}_{\text {control }}\end{array}$ \\
\hline LH-7 & $74 \%$ & 1.73 & 4.23 & 2.45 \\
\hline LH-8 & $64 \%$ & 0.33 & 3.61 & 10.94 \\
\hline LH-9 & $74 \%$ & 0.83 & 8.70 & 10.48 \\
\hline LH-10 & $57 \%$ & 0.76 & 2.34 & 3.08 \\
\hline LH-11 & $78 \%$ & 0.74 & 2.91 & 3.93 \\
\hline
\end{tabular}

Table 4. Effects of tested compounds on LDH activity in culture medium. Cells were treated $24 \mathrm{~h}$ with tested compounds, DMSO (vehicle) and/or Triton X-100. The data are expressed as mean \pm SD for five different hepatocyte cultures. In each culture, LDH activity was measured in triplicates. ${ }^{*}=$ value significantly different from DMSO-treated cells $(\mathrm{p}<0.05) . \%$ of cellular damage was calculated: \% damage $=100 *\left(\mathrm{LDH}_{\text {compound }}-\mathrm{LDH}_{\mathrm{DMSO}}\right) /\left(\mathrm{LDH}_{\text {Triton }}-\mathrm{LDH}_{\mathrm{DMSO}}\right)$

\begin{tabular}{|l|c|}
\hline Compound & LDH $\left[\mu\right.$ kat.L $\left.{ }^{-1}\right]$ \\
\hline A1 $(1 \mu \mathrm{M})$ & $7.3 \pm 7.5$ \\
\hline A1 $(10 \mu \mathrm{M})$ & $2.9 \pm 1.3$ \\
\hline A1 $(50 \mu \mathrm{M})$ & $6.4 \pm 3.9$ \\
\hline A1 $(100 \mu \mathrm{M})$ & $3.6 \pm 1.9$ \\
\hline & \\
\hline A2 $(1 \mu \mathrm{M})$ & $-2.0 \pm 3.2$ \\
\hline A2 $(10 \mu \mathrm{M})$ & $-5.4 \pm 2.2$ \\
\hline A2 $(50 \mu \mathrm{M})$ & $-0.4 \pm 4.2$ \\
\hline A2 $(100 \mu \mathrm{M})$ & $17.6 \pm 5.7^{*}$ \\
\hline & \\
\hline A3 $(1 \mu \mathrm{M})$ & $-4.5 \pm 3.1$ \\
\hline A3 $(10 \mu \mathrm{M})$ & $-2.9 \pm 8.6$ \\
\hline A3 $(50 \mu \mathrm{M})$ & $-2.4 \pm 9.4$ \\
\hline A3 $(100 \mu \mathrm{M})$ & $5.1 \pm 9.2$ \\
\hline & \\
\hline A4 $(1 \mu \mathrm{M})$ & $3.3 \pm 3.3$ \\
\hline A4 $(10 \mu \mathrm{M})$ & $1.9 \pm 7.5$ \\
\hline A4 $(50 \mu \mathrm{M})$ & $2.2 \pm 4.1$ \\
\hline A4 $(100 \mu \mathrm{M})$ & $4.4 \pm 4.5$ \\
\hline & \\
\hline A5 $(1 \mu \mathrm{M})$ & $0.5 \pm 6.5$ \\
\hline A5 $(10 \mu \mathrm{M})$ & $-4.3 \pm 6.9$ \\
\hline A5 $(50 \mu \mathrm{M})$ & $-5.5 \pm 11.1$ \\
\hline A5 $(100 \mu \mathrm{M})$ & $-8.9 \pm 13.6$ \\
\hline & \\
\hline A6 $(1 \mu \mathrm{M})$ & $1.8 \pm 7.5$ \\
\hline A6 $(10 \mu \mathrm{M})$ & $-1.7 \pm 2.0$ \\
\hline A6 $(50 \mu \mathrm{M})$ & $-8.3 \pm 10.2$ \\
\hline A6 $(100 \mu \mathrm{M})$ & $-8.8 \pm 7.4$ \\
\hline
\end{tabular}

\begin{tabular}{|l|c|}
\hline Compound & LDH $\left[\mu\right.$ kat.L $\left.{ }^{-1}\right]$ \\
\hline A7 $(1 \mu \mathrm{M})$ & $1.8 \pm 3.4$ \\
\hline A7 $(10 \mu \mathrm{M})$ & $0.2 \pm 3.2$ \\
\hline A7 $(50 \mu \mathrm{M})$ & $-4.9 \pm 6.5$ \\
\hline A7 $(100 \mu \mathrm{M})$ & $-5.9 \pm 6.8$ \\
\hline & \\
\hline A8 $(1 \mu \mathrm{M})$ & $-0.9 \pm 7.1$ \\
\hline A8 $(10 \mu \mathrm{M})$ & $-2.2 \pm 6.1$ \\
\hline A8 $(50 \mu \mathrm{M})$ & $1.2 \pm 4.3$ \\
\hline A8 $(100 \mu \mathrm{M})$ & $-1.7 \pm 6.6$ \\
\hline & \\
\hline A9 $(1 \mu \mathrm{M})$ & $-2.1 \pm 4.4$ \\
\hline A9 $(10 \mu \mathrm{M})$ & $-2.1 \pm 3.4$ \\
\hline A9 $(50 \mu \mathrm{M})$ & $-7.2 \pm 11.9$ \\
\hline A9 $(100 \mu \mathrm{M})$ & $-8.1 \pm 9.9$ \\
\hline & \\
\hline A10 $(1 \mu \mathrm{M})$ & $0.2 \pm 1.4$ \\
\hline A10 $(10 \mu \mathrm{M})$ & $1.5 \pm 2.6$ \\
\hline A10 $(50 \mu \mathrm{M})$ & $-1.1 \pm 6.1$ \\
\hline A10 $(100 \mu \mathrm{M})$ & $0.1 \pm 9.6$ \\
\hline \multicolumn{2}{|c|}{} \\
\hline A11 $(1 \mu \mathrm{M})$ & $5.7 \pm 1.6$ \\
\hline A11 $(10 \mu \mathrm{M})$ & $6.9 \pm 0.2$ \\
\hline A11 $(50 \mu \mathrm{M})$ & n.d. \\
\hline A11 $(100 \mu \mathrm{M})$ & $-6.2 \pm 11.2$ \\
\hline & \\
\hline A12 $(1 \mu \mathrm{M})$ & $-2.2 \pm 12.0$ \\
\hline A12 $(10 \mu \mathrm{M})$ & $6.3 \pm 1.2$ \\
\hline A12 $(50 \mu \mathrm{M})$ & n.d. \\
\hline A12 $(100 \mu \mathrm{M})$ & $-9.1 \pm 9.2$ \\
\hline
\end{tabular}

\begin{tabular}{|l|c|}
\hline Compound & LDH $\left[\mu\right.$ kat.L $\left.{ }^{-1}\right]$ \\
\hline A13 $(1 \mu \mathrm{M})$ & $-7.5 \pm 7.9$ \\
\hline A13 $(10 \mu \mathrm{M})$ & $17.9 \pm 15.5$ \\
\hline A13 $(50 \mu \mathrm{M})$ & n.d \\
\hline A13 $(100 \mu \mathrm{M})$ & $36.2 \pm 18.5^{*}$ \\
\hline & \\
\hline A14 $(1 \mu \mathrm{M})$ & $5.4 \pm 3.1$ \\
\hline A14 $(10 \mu \mathrm{M})$ & $11.9 \pm 9.5$ \\
\hline A14 $(50 \mu \mathrm{M})$ & n.d \\
\hline A14 $(100 \mu \mathrm{M})$ & $3.1 \pm 5.7$ \\
\hline & \\
\hline A15 $(1 \mu \mathrm{M})$ & $6.5 \pm 0.8$ \\
\hline A15 $(10 \mu \mathrm{M})$ & $-2.6 \pm 8.9$ \\
\hline A15 $(50 \mu \mathrm{M})$ & n.d \\
\hline A15 $(100 \mu \mathrm{M})$ & $9.7 \pm 2.1^{*}$ \\
\hline & \\
\hline A16 $(1 \mu \mathrm{M})$ & $5.7 \pm 0.9$ \\
\hline A16 $(10 \mu \mathrm{M})$ & $6.8 \pm 3.8$ \\
\hline A16 $(50 \mu \mathrm{M})$ & $-6.1 \pm 3.2$ \\
\hline A16 $(100 \mu \mathrm{M})$ & $0.7 \pm 1.0$ \\
\hline & \\
\hline A17 $(1 \mu \mathrm{M})$ & $-7.3 \pm 10.2$ \\
\hline A17 $(10 \mu \mathrm{M})$ & $1.3 \pm 0.1$ \\
\hline A17 $(50 \mu \mathrm{M})$ & $5.6 \pm 4.6$ \\
\hline A17 $(100 \mu \mathrm{M})$ & $-2.8 \pm 3.9$ \\
\hline & \\
\hline A0 $(1 \mu \mathrm{M})$ & $7.5 \pm 1.1$ \\
\hline A0 $(10 \mu \mathrm{M})$ & $9.9 \pm 6.3$ \\
\hline A0 $(50 \mu \mathrm{M})$ & n.d. \\
\hline A0 $(100 \mu \mathrm{M})$ & $23.9 \pm 15.9^{*}$ \\
\hline
\end{tabular}


Table 5. Effects of tested compounds on MTT. Cells were treated $24 \mathrm{~h}$ with tested compounds, DMSO (vehicle) and/or Triton X-100. The data are expressed as mean \pm SD for five different hepatocyte cultures. In each culture, MTT was measured in six parallel samples. ${ }^{*}=$ value significantly different from DMSO-treated cells $(\mathrm{p}<0.05)$. The results are presented as \% of control (DMSO-treated) value. \% of control was calculated: \% of control $=\mathrm{MTT}_{\text {compound }} / \mathrm{MTT}_{\mathrm{DMSO}}$

\begin{tabular}{|l|c|}
\hline Compound & \% of control \\
\hline A1 $(1 \mu \mathrm{M})$ & $94.0 \pm 10.1$ \\
\hline $\mathrm{A} 1(10 \mu \mathrm{M})$ & $89.6 \pm 7.5$ \\
\hline $\mathrm{A} 1(50 \mu \mathrm{M})$ & $93.3 \pm 12.6$ \\
\hline $\mathrm{A} 1(100 \mu \mathrm{M})$ & $92.1 \pm 11.5$ \\
\hline & \\
\hline $\mathrm{A} 2(1 \mu \mathrm{M})$ & $91.8 \pm 10.9$ \\
\hline $\mathrm{A} 2(10 \mu \mathrm{M})$ & $93.3 \pm 15.3$ \\
\hline $\mathrm{A} 2(50 \mu \mathrm{M})$ & $93.7 \pm 5.1$ \\
\hline $\mathrm{A} 2(100 \mu \mathrm{M})$ & $71.0 \pm 16.5^{*}$ \\
\hline & \\
\hline $\mathrm{A} 3(1 \mu \mathrm{M})$ & $96.8 \pm 14.6$ \\
\hline $\mathrm{A} 3(10 \mu \mathrm{M})$ & $92.2 \pm 13.3$ \\
\hline $\mathrm{A} 3(50 \mu \mathrm{M})$ & $94.5 \pm 15.8$ \\
\hline A3 $(100 \mu \mathrm{M})$ & $88.7 \pm 13.3$ \\
\hline & \\
\hline A4 $(1 \mu \mathrm{M})$ & $92.4 \pm 2.9$ \\
\hline A4 $(10 \mu \mathrm{M})$ & $90.6 \pm 8.9$ \\
\hline A4 $(50 \mu \mathrm{M})$ & $95.3 \pm 5.1$ \\
\hline A4 $(100 \mu \mathrm{M})$ & $91.1 \pm 5.7$ \\
\hline
\end{tabular}

\begin{tabular}{|l|c|}
\hline Compound & \% of control \\
\hline A5 $(1 \mu \mathrm{M})$ & $91.8 \pm 12.8$ \\
\hline A5 $(10 \mu \mathrm{M})$ & $94.4 \pm 13.8$ \\
\hline A5 $(50 \mu \mathrm{M})$ & $92.8 \pm 4.3$ \\
\hline A5 $(100 \mu \mathrm{M})$ & $89.2 \pm 9.9$ \\
\hline & \\
\hline A6 $(1 \mu \mathrm{M})$ & $90.9 \pm 11.3$ \\
\hline A6 $(10 \mu \mathrm{M})$ & $92.2 \pm 8.7$ \\
\hline A6 $(50 \mu \mathrm{M})$ & $96.1 \pm 7.7$ \\
\hline A6 $(100 \mu \mathrm{M})$ & $94.5 \pm 12.4$ \\
\hline & \\
\hline A7 $(1 \mu \mathrm{M})$ & $91.4 \pm 4.7$ \\
\hline A7 $(10 \mu \mathrm{M})$ & $89.3 \pm 9.5$ \\
\hline A7 $(50 \mu \mathrm{M})$ & $93.1 \pm 1.5$ \\
\hline A7 $(100 \mu \mathrm{M})$ & $90.6 \pm 3.8$ \\
\hline & \\
\hline A8 $(1 \mu \mathrm{M})$ & $95.5 \pm 10.9$ \\
\hline A8 $(10 \mu \mathrm{M})$ & $92.0 \pm 12.7$ \\
\hline A8 $(50 \mu \mathrm{M})$ & $92.8 \pm 0.2$ \\
\hline A8 $(100 \mu \mathrm{M})$ & $91.8 \pm 7.4$ \\
\hline
\end{tabular}

\begin{tabular}{|l|c|}
\hline Compound & \% of control \\
\hline A9 $(1 \mu \mathrm{M})$ & $96.7 \pm 12.2$ \\
\hline A9 $(10 \mu \mathrm{M})$ & $95.8 \pm 11.7$ \\
\hline A9 $(50 \mu \mathrm{M})$ & $97.3 \pm 9.8$ \\
\hline A9 $(100 \mu \mathrm{M})$ & $98.1 \pm 3.6$ \\
\hline & \\
\hline A10 $(1 \mu \mathrm{M})$ & $92.1 \pm 11.8$ \\
\hline A10 $(10 \mu \mathrm{M})$ & $92.2 \pm 11.6$ \\
\hline A10 $(50 \mu \mathrm{M})$ & $93.5 \pm 10.1$ \\
\hline A10 $(100 \mu \mathrm{M})$ & $91.4 \pm 10.9$ \\
\hline & \\
\hline A16 $(1 \mu \mathrm{M})$ & $89.5 \pm 6.1$ \\
\hline A16 $(10 \mu \mathrm{M})$ & $89.6 \pm 7.4$ \\
\hline A16 $(50 \mu \mathrm{M})$ & $88.8 \pm 3.8$ \\
\hline A16 $(100 \mu \mathrm{M})$ & $84.1 \pm 8.1$ \\
\hline & \\
\hline A17 $(1 \mu \mathrm{M})$ & $90.5 \pm 16.1$ \\
\hline A17 $(10 \mu \mathrm{M})$ & $91.0 \pm 20.5$ \\
\hline A17 $(50 \mu \mathrm{M})$ & $93.8 \pm 10.9$ \\
\hline A17 $(100 \mu \mathrm{M})$ & $96.6 \pm 11.1$ \\
\hline
\end{tabular}

Table 6. Effects of tested compounds on albumin secretion. Cells were treated $24 \mathrm{~h}$ with tested compounds, DMSO (vehicle) and/or Triton X-100. The data are expressed as mean \pm SD for two different hepatocyte cultures. In each culture, albumin secretion was measured in triplicates. ${ }^{*}=$ value significantly different from DMSO-treated cells $(\mathrm{p}<0.05)$. The results are presented as \% of control (DMSO-treated) value. $\%$ of control was calculated: $\%$ of control $=$ ALBUMIN $_{\text {compound }} /$ ALBUMIN $_{\text {DMSO }}$

\begin{tabular}{|c|c|}
\hline Compound & \% of control \\
\hline $\mathrm{A} 0(1 \mu \mathrm{M})$ & $67.5 \pm 5.8^{*}$ \\
\hline $\mathrm{A} 0(10 \mu \mathrm{M})$ & $28.3 \pm 12.6^{*}$ \\
\hline $\mathrm{A} 0(100 \mu \mathrm{M})$ & $17.3 \pm 6.9^{*}$ \\
\hline & \\
\hline $\mathrm{A} 11(1 \mu \mathrm{M})$ & $106.9 \pm 15.5$ \\
\hline $\mathrm{A} 11(10 \mu \mathrm{M})$ & $93.7 \pm 8.8$ \\
\hline $\mathrm{A} 11(100 \mu \mathrm{M})$ & $26.2 \pm 5.7^{*}$ \\
\hline
\end{tabular}

\begin{tabular}{|c|c|}
\hline Compound & \% of control \\
\hline $\mathrm{A} 12(1 \mu \mathrm{M})$ & $154.8 \pm 23.2^{*}$ \\
\hline $\mathrm{A} 12(10 \mu \mathrm{M})$ & $141.1 \pm 34.4^{*}$ \\
\hline $\mathrm{A} 12(100 \mu \mathrm{M})$ & $84.3 \pm 18.8$ \\
\hline & \\
\hline $\mathrm{A} 13(1 \mu \mathrm{M})$ & $74.3 \pm 21.9^{*}$ \\
\hline $\mathrm{A} 13(10 \mu \mathrm{M})$ & $24.6 \pm 7.4^{*}$ \\
\hline $\mathrm{A} 13(100 \mu \mathrm{M})$ & $0.0 \pm 0.0^{*}$ \\
\hline
\end{tabular}

\begin{tabular}{|l|c|}
\hline Compound & \% of control \\
\hline $\mathrm{A} 14(1 \mu \mathrm{M})$ & $93.1 \pm 13.2$ \\
\hline $\mathrm{A} 14(10 \mu \mathrm{M})$ & $85.3 \pm 11.1^{*}$ \\
\hline $\mathrm{A} 14(100 \mu \mathrm{M})$ & $71.1 \pm 8.8^{*}$ \\
\hline & \\
\hline $\mathrm{A} 15(1 \mu \mathrm{M})$ & $86.4 \pm 12.3$ \\
\hline $\mathrm{A} 15(10 \mu \mathrm{M})$ & $80.1 \pm 10.0^{*}$ \\
\hline $\mathrm{A} 15(100 \mu \mathrm{M})$ & $54.4 \pm 9.1^{*}$ \\
\hline
\end{tabular}

polymerization of tubulin, i.e. the essential event in the process of mitosis. Logically, non-proliferating (quiescent) cells, such as human hepatocytes, are much less susceptible to colchicine derivatives than proliferating cells or cell lines.

In contrast, secretion of albumin was a reliable parameter for monitoring the cytotoxicity of the studied compounds. Intact cytoskeleton is essential for albumin secretion even in non-proliferating cells, i.e in human hepatocytes. Colchicine and its derivatives inhibited the secretion of albumin in a dose-dependent manner. These effects were already marked in $1 \mu \mathrm{M}$ concentrations and the inhibition potency correlated with the ability of compounds to inhibit tubulin polymerization (Table 6). For instance $10 \mu \mathrm{M}$ colchicine (A0) decreased albumine secretion down-to $28 \%$ of control value, whereas 10-O-demethylcolchicine (colchiceine; A11) at the same concentration had no effect on albumin secretion. We have previously reported this phenomenon elsewhere ${ }^{3}$.

In conclusion, the biological activity of colchicine and its derivatives is tightly bound to their inhibitory effects on tubulin polymerization. The majority of side effects 
of colchicine is demonstrated in proliferating cells, e.g. intestinal mucosa (nausea, vomiting) or hairs (alopecia) ${ }^{1}$. This implies that replacement of colchicine by derivative with lower anti-tubulin activity will result in lower side effects but also in lower therapeutic effects. However, this is only a hypothesis. Our data show that 10 -O-demethylated derivatives of colchicine (A11, A12) display significantly lower cytotoxicity in human hepatocytes compared to colchicine. In addition, the tested derivatives may have distinct biological activity which is primarily due to interference with microtubules.

\section{ACKNOWLEDGEMENT}

This research was supported by grant MSM 6198959216 from the Ministry of Education, Youth and Sports of the Czech Republic. We thank to MADAUS AG, Cologne, Germany for providing colchicine derivatives.

\section{REFERENCES}

1. Bibas R, Gaspar NK, Ramos-e-Silva M. Colchicine for dermatologic diseases. J Drugs Dermatol 2005; 4:196-204.

2. Ben-Chetrit E, Levy M. Colchicine: 1998 update. Semin Arthritis Rheum 1998; 28:48-59.

3. Dvorak Z, Ulrichova J, Pichard-Garcia L, Modriansky M, Maurel P. Comparative effect of colchicine and colchiceine on cytotoxicity and CYP gene expression in primary human hepatocytes. Toxicol In Vitro 2002; 16:219-27.

4. Jordan A, Hadfield JA, Lawrence NJ, McGown AT. Tubulin as a target for anticancer drugs: agents which interact with the mitotic spindle. Med Res Rev 1998; 18:259-96.
5. Sackett DL, Varma JK. Molecular mechanism of colchicine action: induced local unfolding of beta-tubulin. Biochemistry 1993; 32:13560-5.

6. Modriansky M, Ulrichova J, Dvorak Z. Microtubules and commuting receptors. Letters in Drug Design \& Discovery 2006; 3:567-568.

7. Dvorak Z, Vrzal R, Ulrichova J, Pascussi JM, Maurel P, Modriansky M. Involvement of Cytoskeleton in AhR-Dependent CYP1A1 Expression. Curr Drug Metab 2006; 7:301-3.

8. Dvorak Z, Ulrichova J, Modriansky M. Role of microtubules network in CYP genes expression. Curr Drug Metab 2005; 6:54552.

9. Kosina P, Dvorak Z, Walterova D. [The human hepatocyte: I. A model for studying metabolism and toxicity of xenobiotics]. Ceska Slov Farm 1999; 48:65-71.

10. Maurel $P$. The use of adult human hepatocytes in primary culture and other in vitro systems to investigate drug metabolism in man. Adv Drug Deliv Rev 1996; 22:105-132.

11. Husek A, Sutlupinar N, Sedmera P, Voegelein F, Valka I, Simanek V. Substances from Plants of the Subfamily Wurmbaeoideae and Their Derivatives.104. Alkaloids and Phenolics of ColchicumTurcicum. Phytochemistry 1990; 29:3058-3060.

12. Pichard L, Fabre I, Fabre G, Domergue J, Saint Aubert B, Mourad G, Maurel P. Cyclosporin A drug interactions. Screening for inducers and inhibitors of cytochrome P-450 (cyclosporin A oxidase) in primary cultures of human hepatocytes and in liver microsomes. Drug Metab Dispos 1990; 18:595-606.

13. Isom HC, Secott T, Georgoff I, Woodworth C, Mummaw J. Maintenance of differentiated rat hepatocytes in primary culture. Proc Natl Acad Sci U S A 1985; 82:3252-6.

14. Dvorak Z, Kosina P, Walterova D, Simanek V, Bachleda P, Ulrichova J. Primary cultures of human hepatocytes as a tool in cytotoxicity studies: cell protection against model toxins by flavonolignans obtained from Silybum marianum. Toxicol Lett 2003; 137:201-12.

15. Ulrichova J, Dvorak Z, Vicar J, Lata J, Smrzova J, Sedo A, Simanek V. Cytotoxicity of natural compounds in hepatocyte cell culture models. The case of quaternary benzo[c]phenanthridine alkaloids. Toxicol Lett 2001; 125:125-32. 